\title{
ESTRATÉGIAS TECNOLÓGICAS DE TRÊS MONTADORAS DE MOTORES PARA AUTOMÓVEIS
}

\section{TECHNOLOGICAL STRATEGIES OF THREE CAR ENGINE ASSEMBLERS}

\author{
Aline Lamon Cerra ${ }^{1}$; Jonas Lucio Maia ${ }^{2}$; Alceu Gomes Alves Filho ${ }^{3}$ \\ ${ }^{1}$ Federal University of São Carlos - UFSCar - São Carlos - Brasil alinelc@terra.com.br \\ ${ }^{2}$ Federal University of São Carlos - UFSCar - São Carlos - Brasil jonasmaia@dep.ufscar.br \\ ${ }^{3}$ Federal University of São Carlos - UFSCar - São Carlos - Brasil alceu@power.ufscar.br
}

\begin{abstract}
Resumo
Este artigo tem por objetivo identificar, analisar e comparar, por meio de estudos de caso, as Estratégias Tecnológicas de três montadoras de motores. Duas das montadoras são veteranas (tendo sido aqui instaladas no início da indústria automobilística) e apresentaram ETs muito semelhantes. Constatamos algumas diferenças, por exemplo quanto aos tipos de Capacidades Tecnológicas por elas priorizados elou os esforços realizados em cada tipo de capacidade, mas estas parecem estar mais relacionadas ao ritmo ou aos momentos em que as mudanças são implementadas do que às direções estratégicas escolhidas. Por outro lado, a ET da terceira montadora, a entrante, apresenta diferenças significativas em relação às duas primeiras.
\end{abstract}

Palavras-chave: estratégia tecnológica, capacidades tecnológicas, indústria automobilística.

\section{Introdução}

$\mathrm{Na}$ indústria automobilística global pode-se verificar que, a partir da década de 90 diversas mudanças vêm sendo implementadas em suas cadeias produtivas, com impactos significativos nas relações interfirmas. Dentre elas, tem-se que as grandes montadoras puderam se concentrar em questões mais especializadas em nível de produto, priorizando as atividades e competências relativas à criação de características que identificarão o produto junto ao mercado consumidor, cabendo aos seus fornecedores diretos a responsabilidade sobre o aprimoramento tecnológico dos diferentes sistemas que comporão o veículo (PEREIRA; GEIGER, 2005). Esses fornecedores, por sua vez, repassaram parte de suas atividades para os fornecedores atuantes em níveis inferiores da cadeia de suprimentos.

A indústria automobilística nacional, seguindo a tendência mundial, presenciou, a partir deste período, um novo padrão de relacionamento entre empresas montadoras de veículos e 
autopeças. Com a abertura da economia, diversas montadoras instalaram unidades produtivas em regiões sem tradição no setor automobilístico.

As montadoras que haviam iniciado suas atividades produtivas no início da indústria automobilística brasileira apresentam investimentos em P\&D menos centralizados no exterior e são denominadas "veteranas" por CONSONI (2004). Já as montadoras que aqui se instalaram após os anos 90, apresentam extrema centralização das atividades de engenharia no exterior, sobretudo em relação aos estágios de Desenvolvimento de Produtos (DP), e são denominadas "entrantes" pela mesma autora.

As atividades tecnológicas que podem ser identificadas no Brasil têm sido o resultado do processo de acúmulo de aprendizagem em gerar respostas às demandas do mercado nacional, sendo consideradas como elemento chave para a competição local (CONSONI, 2004).

Neste contexto de competitividade, a literatura sugere a necessidade das empresas empregarem tecnologia estrategicamente, ligando-a a estratégia competitiva da empresa, garantindo assim que tecnologia e estratégia dêem suporte uma à outra, habilitando a empresa para alcançar os melhores resultados em suas metas (DODGSON, 1989; BURGELMAN, MAIDIQUE e WHEELWRIGHT, 1998; ZAHRA, SISODIA e DAS, 1994). A natureza desta ligação é usualmente articulada na Estratégia Tecnológica (ET) da firma.

Este artigo tem por objetivo identificar, analisar e comparar as Estratégias Tecnológicas de três montadoras de motores, sendo duas delas pertencentes ao grupo das veteranas e uma delas ao grupo das montadoras entrantes. Para isso, serão abordadas, em cada empresa, as Capacidades Tecnológicas e as inter-relações entre elas. Em um segundo momento, serão discutidas as diferenças e semelhanças entre as ETs das montadoras, apontando as possíveis causas dessas diferenças e semelhanças e enfatizando as diferenças encontradas entre as montadoras veteranas e a entrante.

A próxima seção trata da literatura de Estratégia Tecnológica, apresentando o conceito de ET que adotamos, e estabelecendo os elementos que serão analisados na prática das empresas. Em seguida, discorre-se a respeito do método da pesquisa e os estudos de caso propriamente ditos. Posteriormente tem-se a análise dos casos e as considerações finais do trabalho.

\section{Estratégia tecnológica}

O conceito de Estratégia Tecnológica tem sido parte da literatura de Gestão de Tecnologia desde o final dos anos 70, mas não se tornou uma área distinta de interesse acadêmico e gerencial até os anos 80 (DODGSON, 1991; DREJER, 1996; FRIAR e HORWITCH, 1985; JONES e SMITH, 1997; KANTROW, 1980; PAVITT, 1990) apud DAVENPORT, CAMPBELL-HUNT e 


\section{SOLOMON (2003).}

Não há ainda um conceito consagrado de ET. GIBBONS e O`CONNER (2003) definem Estratégia Tecnológica como um conjunto de escolhas que a firma faz sobre o estado e qualidade do know-how incorporado a projeto, desenvolvimento e produção de seus produtos e/ou serviços.

Para SOLOMON (2001) apud DAVENPORT, CAMPBELL-HUNT e SOLOMON (2003), a Estratégia Tecnológica abrange a aquisição, gestão e exploração de conhecimentos e recursos tecnológicos da organização para alcançar suas metas de negócios.

Neste trabalho, entendemos a Estratégia Tecnológica como um conjunto de esforços da empresa que visa aumentar suas capacidades tecnológicas e implementar mudanças, seja nos sistemas produtivos, seja em produtos e processos:

A Estratégia Tecnológica diz respeito a um conjunto de esforços e ações da empresa no sentido de ampliar sua capacidade tecnológica, tanto no âmbito das atividades de P\&D como nas demais áreas da empresa, para a implementação da mudança técnica. Engloba esta última a criação de novas técnicas e mudanças não necessariamente novas nos elementos que compõem e caracterizam um sistema de produção, seja do ponto de vista da empresa, seja em relação às fronteiras internacionais (ALVES FILHO, 1991).

Adotamos o conceito de ALVES FILHO (1991) que utiliza o termo mudança técnica para se referir às mudanças nos elementos que compõem e caracterizam um sistema de produção. PAVITT e BELL (1993) definem a mudança técnica como sendo a introdução de tecnologias em novos produtos ou processos através de investimentos, ou ainda, adaptação incremental e melhoria da capacidade de produção existente.

Quanto à capacidade tecnológica, BELL e PAVITT (1993) a definem como os "recursos necessários para gerar e administrar mudanças técnicas, incluindo habilidades, conhecimentos e experiências e estruturas e links institucionais". A mudança técnica, segundo estes mesmos autores trata-se de qualquer maneira na qual uma nova tecnologia é incorporada dentro da capacidade de produção de firmas e economias.

DAHLMAN, ROSS-LARSON e WESTPHAL (1987), WESTPHAL, KIM e DAHLMAN (1985) apud ALVES FILHO (1991) sugerem três tipos de capacidades abarcadas pela ET:

- Capacidade de Inovação: para criar novas tecnologias, desenvolver novos produtos ou serviços. Trata-se da capacidade para introduzir mudanças técnicas (radicais ou incrementais).

- Capacidade de Investimento: para adquirir tecnologia, para substituir, expandir e implantar instalações, adaptadas às condições de investimento;

- Capacidade de Produção e adaptação: para operar as instalações produtivas, tendo em vista a obtenção de eficiência produtiva; abrange, além da produção, as possíveis adaptações da tecnologia. 
FORD (1989) sugere uma quarta capacidade, a Capacidade de Exploração e Proteção de Tecnologias, para vender ou prestar serviços no mercado a partir de tecnologias dominadas e também para implementar medidas que impeçam que conhecimentos adquiridos sejam copiados por concorrentes.

As fronteiras entre esses quatro tipos de capacidade tecnológica não são claras, mas é certo que investimentos e esforços específicos são requeridos para cada tipo de capacidade (WESTPHAL, KIM e DAHLMAN (1985) apud ALVES FILHO (1991)).

\section{Método de pesquisa}

O trabalho se estrutura basicamente em duas partes: revisão bibliográfica acerca dos tópicos associados ao tema e pesquisa de campo. A pesquisa de campo foi realizada por meio de estudos de caso, e portanto pode ser caracterizada como qualitativa e descritiva (ou exploratória).

O método de estudo de caso é apropriado à investigação de um fenômeno contemporâneo dentro de seu contexto real, por meio de múltiplas fontes de evidência: entrevistas, observações, documentos etc (YIN, 1989 apud LAZZARINI, 1997). Para o autor, a possibilidade de se utilizar várias fontes de evidência é considerada uma das particularidades (e vantagens) da pesquisa baseada em estudos de caso.

Foram então realizados estudos de caso nas três montadoras escolhidas, fundamentados em entrevistas semi-estruturadas que duraram cerca de uma hora cada. Nas empresas, as pessoas entrevistadas serão preferencialmente aquelas ligadas à tecnologia de produtos, processos e gestão, ou seja, diretores ligados à área tecnológica.

\section{Estudos de caso}

De modo a não identificar as empresas, as montadoras são tratadas como A, B e C, apresentadas, respectivamente, a seguir:

Montadora A: Seus principais produtos são motores $1.0 \mathrm{cc}$ e 1.4, parte deles exportada, e motores 1.6 para veículos do mercado brasileiro. Este conjunto de motores compreende 36 modelos do produto, envolvendo três linhas de produção.

Vale ressaltar que um dos principais aspectos valorizados pela montadora de veículos é a flexibilidade, tanto a flexibilidade de volume (capacidade de responder a qualquer quantidade requerida) como também a flexibilidade de mix de produção (capacidade de aumentar a diversidade de produtos, de modo a adequar-se às necessidades do cliente).

A busca por redução de custos ganhou relevância para a planta de motores nos últimos anos, 
quando esta passou a considerar extremamente importante priorizar custos de produção (custo total do produto vendido), custo de materiais diretos e custos indiretos (administração, manutenção etc). De certo modo, esta priorização também foi influenciada por medidas impostas pelas montadoras de veículos após a realização de grandes investimentos e a não obtenção dos retornos esperados.

Montadora B: Existem basicamente duas famílias de motores, ambas foco deste estudo:

- Família 1: motores de baixa e média cilindradas (1.0 cc, 1.4, 1.6 e 1.8).

- Família 2: motores de alta cilindrada (1.8, 2,0, 2.2 e 2.4).

A partir do ano 2000 a empresa realizou investimentos em suas linhas de produção, com o objetivo de aumentar a capacidade produtiva; para isso, a montadora adquiriu novas máquinas e equipamentos.

Atualmente, diversificar produtos (flexibilidade de mix) é o aspecto mais valorizado pela montadora, que faz internamente a fundição de alguns componentes para motores.

Montadora C: Nascida a partir de uma joint venture firmada em 1996 entre uma empresa alemã do setor automobilístico e uma outra de origem americana, a Montadora C é resultado de um investimento de US\$ 500 milhões em cotas iguais de participação.

O primeiro motor (1.6) foi fabricado em 1999. Atualmente montadora produz motores a gasolina 1.4 e 1.6 litro, ambos com 16 válvulas, compreendendo 23 modelos de motores. Tais motores são destinados ao mercado externo por via marítima.

Os equipamentos utilizados nas linhas como máquinas com comando numérico e dedicadas são novos, mas não são flexíveis, pois somente permitem a montagem dos dois tipos de motores da empresa (1.4 e 1.6).

Deste modo, diferentemente das Montadoras A e B, flexibilidade não é um dos aspectos mais valorizados pela empresa. Nesta, qualidade e reduções de custos parecem prevalecer em relação às demais prioridades competitivas. Isso se justifica pelo fato da montadora operar com capacidade ociosa, canalizando suas iniciativas para a qualidade e custo.

Em 2006 a empresa viveu um período que chamou de "transição". Em julho de 2007 deverá ter sido terminado o prazo pré-estabelecido para duração da joint venture e ocorrido a saída programada da empresa alemã; a empresa americana permanecerá, por tempo indeterminado.

A tabela a seguir sintetiza, de modo comparativo, as ETs das montadoras, que em seguida são discutidas. Vale ressaltar que as Capacidades de Exploração e Proteção não estão citadas, pois nenhuma das montadoras realiza esforços voltados a elas. 
Para obter maior flexibilidade de mix de produtos e de volume de produção (gerar Mudanças Técnicas em produtos e processos), a

Estratégia Montadora A tem buscado Tecnológica ampliar suas capacidades de Inovação, Investimento e de Produção e Adaptação. As Mudanças Técnicas em processos ocorrem em função da introdução de novos produtos e também para gerar melhorias nas linhas que já vêm operando.
Para obter maior flexibilidade de mix de produtos (gerar Mudanças Técnicas em produtos e processos), a Montadora B tem buscado ampliar sua Capacidade de Inovação e de Investimento. Mudanças Técnicas em processos estão, na maioria dos casos, condicionadas à introdução de novos modelos de produtos nas linhas de produção, ou seja, às Mudanças Técnicas em Produtos.
Para obter autonomia tecnológica para realizar atividades locais de DP, a Montadora $\mathrm{C}$ tem se voltado às capacidades de Inovação e Investimento. Mudanças Técnicas em processos estão, na maioria dos casos, condicionadas à introdução de novos produtos (Mudanças Técnicas em produtos).

\section{Capacidade de Inovação}

Pretende diversificar os modelos de motores e para isso reestruturou o setor responsável pelas atividades de desenvolvimento de produtos/processos. Realocou pessoas e colocou um coordenador responsável por cada projeto.
Pretende diversificar os modelos de motores e para isso ampliou o setor responsável pelas atividades de desenvolvimento de produtos/ processos. $\mathrm{O}$ número de pessoas envolvidas aumentou consideravelmente (de 150 para 200 pessoas).
Pretende dar inicio às atividades locais de desenvolvimento de produtos e para isso conta com o apoio da matriz. Os engenheiros responsáveis pelas atividades locais de DP estão sendo treinados no Centro Tecnológico da matriz no exterior.
A busca por novas tecnologias junto à matriz estrangeira não parece ocorrer com a mesma intensidade de

Capacidade de Investimento

ocorre. Ainda é intensa a

troca de conhecimentos com a

matriz e os novos projetos

desenvolvidos localmente precisam passar pelo seu aval. Outra fonte de conhecimentos são os fornecedores, apesar de a montadora deter a tecnologia essencial de componentes importantes e da montagem dos motores.

A busca por conhecimentos tecnológicos na matriz é

Idem a Montadora A. intensa. Há pouca autonomia tecnológica local e esforços no sentido de ampliá-la estão sendo realizados.

$\mathrm{Na}$ fábrica foi criada uma estrutura composta por sete

Capacidade de engenheiros que são ligados Produção e aos lideres das linhas de 


\begin{tabular}{|c|c|c|c|}
\hline Adaptação & $\begin{array}{l}\text { Produção e à Engenharia de } \\
\text { Produtos. A planta passou a } \\
\text { ter maior autonomia para } \\
\text { planejar mudanças em } \\
\text { processos de produção e } \\
\text { também para implementá-las. }\end{array}$ & & \\
\hline $\begin{array}{l}\text { Mudanças } \\
\text { Técnicas em } \\
\text { produtos }\end{array}$ & $\begin{array}{l}\text { Novos modelos de motores } \\
\text { são desenvolvidos } \\
\text { localmente, a partir de } \\
\text { modelos já existentes. }\end{array}$ & Idem a Montadora A. & $\ldots$ \\
\hline $\begin{array}{c}\text { Mudanças } \\
\text { Técnicas em } \\
\text { processos de } \\
\text { produção }\end{array}$ & $\begin{array}{l}\text { Podem ocorrer adaptações } \\
\text { nos processos quando um } \\
\text { novo modelo de motor vai ser } \\
\text { fabricado e também podem } \\
\text { ocorrer depois que um } \\
\text { modelo esta sendo fabricado; } \\
\text { neste ultimo caso, as } \\
\text { mudanças visam melhorias } \\
\text { nos processos. }\end{array}$ & $\begin{array}{l}\text { Na maior parte dos casos } \\
\text { ocorrem inovações } \\
\text { incrementais/adaptações nos } \\
\text { processos quando um novo } \\
\text { modelo de motor vai ser } \\
\text { fabricado. }\end{array}$ & Idem a Montadora B. \\
\hline
\end{tabular}

\section{Análises dos casos}

Quando as atividades das subsidiárias veteranas se iniciaram no Brasil, houve uma tentativa de padronização do design. No entanto, adaptações locais foram necessárias, dada as preferências dos consumidores locais por veículos de baixo custo, as diferentes condições dos combustíveis e das estradas, as características de materiais locais e especificações em relação a regras locais em segurança e poluição.

Tais adaptações propiciaram o início das atividades locais de Desenvolvimento de Produtos. Desse modo, os conhecimentos tecnológicos acumulados no Brasil estavam limitados a esforços localizados de adaptação dos produtos e dos processos de manufatura às condições locais de mercados e insumos, e que se sustentavam a partir da incorporação de tecnologias geradas no exterior.

Essas empresas foram, com o passar do tempo, ampliando as atividades da engenharia local, embora mantendo relações com os centros de pesquisas das matrizes.

As atividades de desenvolvimento de produtos e/ou processos, nas três montadoras estudadas, são conduzidas por uma equipe multifuncional de pessoas da empresa, que representam as diversas áreas (além das pessoas da Engenharia, envolve representante (s) da Produção, Qualidade, Compras, Marketing etc).

As duas montadoras veteranas iniciaram os investimentos em atividades de engenharia de produto no Brasil em períodos diferentes, sendo a Montadora B a pioneira delas, e realizaram desenvolvimentos locais importantes, especialmente em motores de baixas cilindradas e de 
combustíveis flexíveis. Vale ressaltar que a Montadora A foi a pioneira no lançamento de motores 1000 cc e bi-combustíveis.

Ao compararmos o departamento voltado às atividades tecnológicas no Brasil de cada uma das veteranas estudadas podemos observar que, embora na Montadora B haja um número maior de pessoas envolvidas, estas são mais qualificadas na Montadora $\mathrm{A}$, onde encontramos um número mais elevado de pessoas que cursaram graduação e pós-graduação. Este fato pode em parte justificar o pioneirismo da Montadora A no lançamento de novos modelos de motores.

De modo muito semelhante, as montadoras A e B apresentam autonomia tecnológica crescente para desenvolver localmente novos modelos de motores a partir de outros já existentes (Mudanças Técnicas em produtos) e realizar as respectivas mudanças nos processos de produção (Mudanças Técnicas em processos).

Considerando que este estudo trata das características recentes das empresas, observamos que atualmente a Montadora A está buscando obter maior flexibilidade de mix de produtos e de volume de produção; já a Montadora $\mathrm{B}$, tendo realizado há tempos atrás investimentos em equipamentos que a levaram à maior capacidade produtiva, tem focado seus esforços na diversificação dos produtos (flexibilidade de mix) para manter-se competitiva no mercado brasileiro.

Buscando diversificar os modelos de motores, ambas estão direcionando esforços para ampliar a Capacidade de Inovação. Enquanto a Montadora A reestruturou o setor destinado às atividades de DP, a Montadora B aumentou o número de pessoas envolvidas.

Essas montadoras, no que diz respeito à Capacidade de Investimento, além de manterem suas respectivas matrizes como importantes fontes de conhecimentos, conforme já mencionado, também encontram nas cadeias de suprimentos empresas que dominam a tecnologia de componentes importantes para motores. No entanto, as montadoras definem as normas de aprovação de cada componente e dominam a aplicação desses no produto final, ou seja, controlam as interfaces e o conhecimento de como unir os diferentes componentes em um produto com funcionalidade integrada (montagem dos motores).

Apenas a Montadora A demonstrou esforços atualmente no que se refere à Capacidade de Produção e Adaptação. Com a criação de uma estrutura na fábrica composta por engenheiros ligados aos lideres das linhas de Produção e à Engenharia de Produtos, a planta passou a ter maior autonomia para planejar mudanças em processos de produção e também para implementá-las. Essa estrutura auxiliou a montadora a realizar as mudanças nos processos necessárias à introdução dos novos modelos de produtos na fábrica, ao mesmo tempo para obter maior flexibilidade quanto ao volume de produção e gerar melhorias que visam economias.

$\mathrm{Na}$ Montadora B as mudanças em processos estão, na maioria dos casos, relacionadas aos 
novos modelos de motores introduzidos.

Nenhuma das montadoras explora tecnologias desenvolvidas por outras empresas para incorporar em seus próprios produtos e processos, como também não exploram (vendem) tecnologias por elas desenvolvidas. Além de não focalizarem a Capacidade de Exploração, também não têm nenhum programa sistematizado que vise a proteção dos conhecimentos acumulados.

A Montadora C, por outro lado, diferencia-se significativamente das duas primeiras - a matriz da montadora entrante possui diferente estratégia de divisão e localização de atividades de desenvolvimento tecnológico e de projeto de produto com relação a subsidiaria brasileira.

No Brasil há um setor que conta com sete engenheiros responsáveis pelas atividades tecnológicas; estas consistem em adaptar os processos de produção existentes na planta aos novos modelos de motores que são desenvolvidos na matriz. A partir do ano de 2006, no entanto, a Montadora C passou a ter como meta criar no Brasil uma "Engenharia de Produto". Neste contexto, a ET da empresa enfatiza as capacidades de Inovação e Investimento.

As pessoas que exercem as atividades tecnológicas locais, que já eram bastante integradas com a engenharia da matriz, intensificaram os contatos e as trocas de conhecimentos. Estes engenheiros brasileiros estão passando por treinamentos no exterior e pretendem, em um futuro próximo (2007), começar a desenvolver novos modelos de motores, a partir daqueles que vêm sendo aqui fabricado.

Desse modo, as Mudanças Técnicas em produtos continuam sendo realizadas na matriz. A subsidiária recebe o design do produto e os adapta aos processos de produção vigentes (Mudanças Técnicas em processo). Mesmo as atividades tecnológicas voltadas aos processos são relativamente raras já que os processos não são flexíveis e apenas dois modelos de motores são produzidos.

Comparando as montadoras veteranas com a entrante, podemos dizer que diferentes Estratégias Tecnológicas levam a padrões (direções e ritmos) de Mudanças Técnicas diferentes.

As atividades tecnológicas das montadoras, em grande medida, orientam as atividades de DP dos fornecedores, já que estas, quando decidem lançar um novo modelo de motor, repassam à cadeia de suprimentos a demanda por novos componentes. Em alguns casos, as montadoras passam especificações para os fornecedores desenvolverem componentes de acordo com esse novo motor, mas há também casos em que as montadoras cedem o design dos produtos para os fornecedores e esses se responsabilizam pelo design do processo de produção. Há ainda casos em que a montadora passa aos fornecedores todo o know-how relativo ao produto e ao processo, e eles se responsabilizam pela produção do componente em questão. 


\section{Considerações finais}

Os temas relacionados à tecnologia e à gestão de tecnologia ganharam relevância nos últimos anos, quando a tecnologia passou a ser entendida como fator crucial de competitividade em diversos setores econômicos, e pode-se constatar que a literatura sobre tais temas tornou-se mais rica e densa.

Este trabalho apresenta e compara as ETs de três montadoras de motores para automóveis instaladas no Brasil em diferentes períodos, considerando suas capacidades tecnológicas e as mudanças técnicas geradas em seus produtos e processos de produção.

Os resultados mostram que embora essas montadoras externalizem grande parte de suas atividades, sobretudo em DP para fornecedores, o controle das interfaces e o conhecimento de como unir os diferentes módulos e componentes em um produto com funcionalidade integrada ainda pertence à montadora. O domínio desse conhecimento faz com que a capacidade de inovar no mercado brasileiro de automóveis ainda pertença à montadora.

As duas montadoras veteranas apresentaram ETs muito semelhantes, com autonomia tecnológica crescente em relação às suas respectivas matrizes para realizar atividades de DP. Constatamos algumas diferenças, por exemplo quanto aos tipos de Capacidades Tecnológicas por elas priorizados e/ou os esforços realizados em cada tipo de capacidade, mas estas parecem estar mais relacionadas ao ritmo ou aos momentos em que as mudanças são implementadas do que às direções estratégicas escolhidas. Por outro lado, a ET da montadora entrante apresenta diferenças significantes em relação às duas primeiras.

A montadora $\mathrm{C}$ apresenta um setor dedicado a atividades tecnológicas (relacionadas a desenvolvimento de processos, já que os produtos são desenvolvidos na matriz) significativamente inferior ao das veteranas no que se refere ao número de pessoas envolvidas. As atividades realizadas também são menos freqüentes, mesmo se tratando de processos, pois estes últimos não são flexíveis. Por outro lado, a flexibilidade (de mix de produtos e de volume de produção) são os aspectos mais valorizados pelas veteranas. As montadoras atribuem a seus fornecedores de primeira camada parte da responsabilidade em aumentar a flexibilidade e coordenar tal aumento nas camadas inferiores dos fornecedores.

Vale ressaltar que a montadora entrante aqui estudada se diferencia bastante das outras montadoras deste grupo. Enquanto esta apresenta um mix de motores reduzido, voltado especificamente para o mercado externo, as outras montadoras (entrantes), de acordo com a pesquisa de Alves Filho et al (2006), apresentam um mix de produtos relativamente pequeno e dirigido aos mesmos segmentos do mercado brasileiro e do Mercosul.

Os resultados deste estudo nos permite dizer que diferentes ETs levam a diferentes 
Mudanças Técnicas (em produtos e processos).

Trabalhos futuros poderiam ser realizados estudando as ETs de todas as montadoras de motores instaladas no Brasil e os esforços que estas realizam em conjunto com seus fornecedores de componentes.

\begin{abstract}
This paper aims at identifying, analyzing and comparing, by means of case studies, the Technological Strategies (TS) of three car engine assemblers. Two of them can be considered "mature companies" (they set up their production plants in Brazil at the beginning of national automotive industry) and portray similar TSs. Some differences could be identified, for example, in the sorts of Technological Capabilities they have prioritized and/or the efforts made in each capability, but such differences are more related to the pace or to the moments at which technological changes were implemented, than to strategic directions deliberately chosen. On the other hand, the TS undertaken by the third assembler, a newcomer (set up in Brazil after the 90's), presents a very high centralization of engineering activities in the international headquarters and meaningful differences when compared to the two other companies.
\end{abstract}

Keywords: technological strategies, technological capabilities, automotive industry.

\title{
Referências
}

ALVES FILHO, A.G.; CERRA, A.L.; NOGUEIRA, E.; MAIA, J.; MARTINS, M.F.; BONADIO, P.V.G.; BENTO, P.E.G.; RESENDE, R.A.; MARTINS, R.A.; VANALLE, R.M. Estratégias de Produção nas unidades produtoras de motores para automóveis. Relatório Final (Processo CNPq 477515/03-7).162p. 2006.

ALVES FILHO, A.G. Estratégia tecnológica, desempenho e mudança: estudo de caso em empresas da indústria de calçados. 1991. Tese (Doutorado em Engenharia de Produção) - Escola Politécnica, Universidade de São Paulo. São Paulo.

BELL, R. M.; PAVITT, K. Technological accumulation and industrial growth: contrasts between developed and developing countries. Industrial and Corporate Change, p.157 - 210, 1993.

BURGELMAN, R. A.; MAIDIQUE, M. A.; WHEELWRIGHT, S. C. Strategic management of technology and innovation. 2nd edition, Mc Graw-Hill, 1998.

CONSONI, F.L. Da Tropicalização ao projeto de veículos: um estudo das competências em desenvolvimento de produtos nas montadoras de automóveis no Brasil. 2004. Tese (Doutorado em Política Científica e Tecnológica) Instituto de Geociências - Universidade Estadual de Campinas. 269 p. Campinas - SP.

DAVENPORT, S.; CAMPBELL-HUNT, C.; SOLOMON, J. The dynamics of Technology Strategy: an exploratory study. R\&D Management, V.33, n.5, 2003.

cross ${ }^{\text {ret }}$

DODGSON, M. Technology Strategy and the Firm: management and public policy. Longman Group UK, 1989.

FORD, D. Develop your strategy. Engineering Management Review, v.17, n.3, p.16-26, Sept., 1989.

GIBBONS, P.T.; O'CONNOR, T. Strategic posture, technological strategy and performance. Journal of Enterprising Culture. V.11, n.2, june 2003. p.131-146.

cross ${ }^{\text {ref }}$

LAZZARINI, S. G. Estudos de caso: aplicações e limites do método. In: FARINA, E. Estudos de caso em agribusiness. São Paulo: Pioneira, 1997. 
PEREIRA, G.M.; GEIGER, A. Complexidade do produto e volume de produção como determinantes da estratégia de desenvolvimento de fornecedores automotivos. Gestão \& Produção. V. 12, n.2, p.191-201, maio-ago 2005.

ZAHRA, S. A.; SISODIA, R. S.; DAS, S. R. Technological choices within competitive strategy types: a conceptual integration. International Journal Technology Management. v.9, n.2, 1994.

\section{Dados dos autores:}

Nome completo: Aline Lamon Cerra

Filiação institucional: Universidade Federal de São Carlos -UFSCar

Filiação institucional: Departamento de Engenharia de Produção

Função ou cargo ocupado: Pós-doutoranda

Endereço completo: Rodovia Washington Luis (SP-310) Km 235 Monjolinho

São Carlos - SP, CEP: 13565-905

Telefones para contato: (16) 33518236 Ramal 9223

E-mail:alinelc@terra.com.br

Nome completo: Jonas Lucio Maia

Filiação institucional: Universidade Federal de São Carlos - UFSCar

Departamento de Engenharia de Produção

Função ou cargo ocupado: Doutorando

Endereço completo: Rodovia Washington Luis (SP-310) Km 235 Monjolinho

São Carlos - SP, CEP: 13565-905

Telefones para contato: (16) 33518236 Ramal 9223

E-mail:jonasmaia@dep.ufscar.br

Nome completo: Alceu Gomes Alves Filho

Filiação institucional: Universidade Federal de São Carlos - UFSCar

Departamento de Engenharia de Produção

Função ou cargo ocupado: Professor

Endereço completo: Rodovia Washington Luis (SP-310), Km 235, Monjolinho

São Carlos - SP, CEP: 13565-905

(16) 33518236 Ramal 9223

E-mail:alceu@power.ufscar.br 